\title{
Praceadiago of SPIE
}

Third Order Nonlinear Optical Properties of Stacked Bacteriochlorophylls in Bacterial Photosynthetic Light-harvesting Proteins

\author{
Alis 12997 \\ 051 \\ L. X. Chen ${ }^{a}$, Philip D. Laible ${ }^{a}$, Frank C. Spano ${ }^{b}$, Eric S. Manas ${ }^{b}$
}

${ }^{a}$ Chemistry Division, Argonne National Laboratory, Argonne, Illinois 60439

${ }^{b}$ Department of Chemistry, Temple University, Philadelphia, Pennsylvania 19122

\section{ABSTRACT}

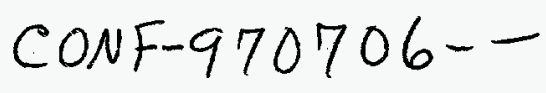

Enhancement of the nonresonant second order molecular hyperpolarizabilities $\gamma$ were observed in stacked macrocyclic molecular systems, previously in a $\mu$-oxo silicon phthalocyanine (SiPcO) monomer, dimer and trimer series, and now in bacteriochlorophyll $a$ (BChla) arrays of light harvesting (LH) proteins. Compared to monomeric BChla in a tetrahydrofuran (THF) solution, the $\langle\gamma\rangle$ for each macrocycle was enhanced in naturally occurring stacked macrocyclic molecular systems in the bacterial photosynthetic LH proteins where BChla's are arranged in tilted face-to-face arrays. In addition, the $\gamma$ enhancement is more significant in B875 of LH1 than in B850 in LH2. Theoretical modeling of the nonresonant $\gamma$ enhancement using simplified molecular orbitals for model SiPcO indicated that the energy level of the two photon state is crucial to the $\gamma$ enhancement when a two photon process is involved, whereas the charge transfer between the monomers is largely responsible when one photon near resonant process is involved. The calculated results can be extended to $\gamma$ enhancement in B875 and B850 arrays, suggesting that BChla in B875 are more strongly coupled than in B850. In addition, a 50-160 fold increase in $\langle\gamma\rangle$ for the $S_{1}$ excited state of relative to $S_{0}$ of bacteriochlorophyll in vivo was observed which provides an alternative method for probing excited state dynamics and a potential application for molecular switching.

Key words: phthalocyanine, bacteriochlorophyll, light harvesting protein, third order nonlinear optical response, degenerate four wave mixing

\section{INTRODUCTION}

The enhancement of the third order nonlinear optical susceptibility $\chi^{(3)}$ as a function of number of $\pi$ electrons in a linear or a planar molecule has been observed and can be explained as a result of conjugated $\pi$-electron delocalization on the same plane ${ }^{1-3}$. However, the effect of $\pi-\pi$ electron interactions between different $\pi$-conjugated structures on $\chi^{(3)}$ is not well understood.

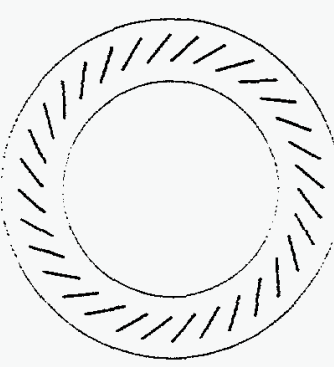

LH1

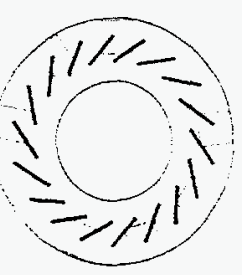

$\mathrm{LH} 2$

Figure 1 Graphic representations of the arrangement of BChla pigments in $\mathrm{LH}$ proteins based on known or proposed structures for the membrane-bound, pigment-protein complexes ${ }^{6.7}$.

Such a $\pi-\pi$ electron interaction differs from the cases of in-plane oneand two-dimensional conjugated systems because the $\pi-\pi$ electrons do not have direct covalent bonding and the $\pi-\pi$ interaction occurs in a direction that is perpendicular to the $\pi$-electron conjugation plane. This kind of interaction often results from stacking $\pi$-conjugated molecules, such as organic crystals of $\pi$-conjugated systems, ordered liquid crystals of macrocycles, Langmuir-Blogett films, and BChla in bacterial photosynthetic reaction centers and light harvesting proteins.

We have previously measured the second hyperpolarizabilities $\langle\gamma\rangle$ of a perfect "face-to-face" stacked macrocycle system, $\mu$-oxo silicon phthalocyanine $(\mathrm{SiPcO})_{n}(\mathrm{n}=1-3)$ using nonresonant degenerate four wave mixing (DFWM) at $1064 \mathrm{~nm}^{4}$. According to our results, $<\gamma>$ value for each monomer in this system is greatly enhanced as the number of monomer units in the oligomer increasest. We now extend our study to naturally occurring "face-toface" macrocycle aggregates, BChla arrays in bacterial light 


\section{DISCLAIMER}

This report was prepared as an account of work sponsored by an agency of the United States Government. Neither the United States Government nor any agency thereof, nor any of their employees, makes any warranty, express or implied, or assumes any legal liability or responsibility for the accuracy, completeness, or usefulness of any information, apparatus, product, or process disclosed, or represents that its use would not infringe privately owned rights. Reference herein to any specific commercial product, process, or service by trade name, trademark, manufacturer, or otherwise does not necessarily constitute or imply its endorsement, recommendation, or favoring by the United States Government or any agency thereof. The views and opinions of authors expressed herein do not necessarily state or reflect those of the United States Government or any agency thereof. 


\section{DESCLAMIER}

Portions of this docament moy be Illepibie in electronic iroge produets Images are produced from the best araitable origion doenomeat 
harvesting complexes LH1 and LH2. The $\langle\gamma\rangle$ values for each BChla unit in monomeric BChla solution, in LH1 and LH2 arrays are investigated. We also carried out a semi-empirical $\gamma$ calculation on the "face-to-face" $(\mathrm{SiPcO})_{n}$ system, reproducing the trend of the $\langle\gamma\rangle$ as a function of monomer numbers in the system ${ }^{5}$. The purpose of this study is to correlate the third order nonlinear optical response with the electronic interactions between the macrocycles as well as photoinduced energy or electron transfer processes in these systems.

Tilted "face-to-face" BChla arrays have been discovered for both LH1(Rhodospirillum rubrum) and LH2 (Rhodopseudomonas acidophila) ${ }^{7}$ complexes (see Figure 1). LH2 contains two BChla arrays, B800 and B850, and LH1, one array, B875. B800 is a BChla array of nine monomeric bacteriochlorophylls with a side-by-side arrangement, and B850 is an 18-BChla array with tilted "face-to-face" configuration ${ }^{6}$. B875 of LH1 has a configuration similar to B850 of LH27. BChla arrays in B850 and B875 are bound in membrane spanning $\alpha$-helical peptides in such a way that they are simultaneously Jaggregates in one direction and $\mathrm{H}$-aggregates in another ${ }^{8}$. The configurations of the $\mathrm{BChl} a$ arrays as well as their interaction with the protein matrices tune the energies of the different $\mathrm{BChla}$ arrays in such a way that allows a ultrafast energy flow (energetically-downhill) from the outer LH2 to inner LH1 and then to the reaction center (RC) where the initial electron transfer reaction occurs'.

Moreover, the excited state $\langle\gamma\rangle$ of BChla in LH1 and LH2 were also measured. In these cases, the reference state is the excited state $S_{1}$ populated by exciting molecules from the ground state $S_{0}$. Although whether $\langle\gamma\rangle$ is still in non-resonant regime is not clear, we have observed dramatic $\langle\gamma\rangle$ enhancement relative to the ground state for BChla in LH1, similar to the excitation enhancement observed before in dipenylhexatriene (DPH) solution ${ }^{10}$.

\section{EXPERIMENTAL}

\section{a. Samples}

Preparation of $\mathrm{BChl} a$ and $\mathrm{LH}$ protein samples was described previously" ${ }^{11}$. The detergents used in the final solutions for LH1 and LH2 are n-octyl- $\beta$-D-glucoside ( $\beta O \mathrm{OG} ; 0.8 \%$ ) and N,N-dimethyldodecylamine-N-oxide (LDAO; $1 \%$ ), respectively. Both samples were buffered by $10 \mathrm{mM}$ Tris at $\mathrm{pH}=7.5$. Concentrations of $\mathrm{BChl} a$ in the $\mathrm{LH} 2$ samples were determined based on the extinction coefficient for BChla at $850 \mathrm{~nm}, 184 \mathrm{mM}^{-1} \mathrm{~cm}^{-112}$, and the molar ratio of BChl $a$ in $\mathrm{B} 800$ and in B850 $0^{6}$. The $\epsilon_{800}$ for BChla in $\mathrm{B} 800$ is $240 \mathrm{mM}^{-1} \mathrm{~cm}^{-1}$ based on our calculation. An $\epsilon_{880}$ of $140 \mathrm{mM} \mathrm{tm}{ }^{-1}$ for BChla at $875 \mathrm{~nm}$ was used to determine the concentration of $\mathrm{BChl} a$ in the LH1 sample ${ }^{13}$.

\section{b. DFWM and Excited State DFWM Measurements}

The laser system for ground state DFWM measurements was described elsewhere $e^{11}$. The $\chi^{(3)}$ measurements were carried out with polarization directions of all four laser beams aligned vertically in the laboratory frame. The excited state DFWM mixing experiment was conducted by introducing an excitation laser pulse to the previous DFWM setup. The detail of the experimental setup was described previously ${ }^{11}$. While three $1064 \mathrm{~nm}$ pulses (the two pumps and one probe) were overlapped in time and space, the excitation pulse was delayed relative to the $1064 \mathrm{~nm}$ pulses. The resulting phase conjugated signal as a function of the delay of the $583 \mathrm{~nm}$ excitation pulse was then collected. Details of the femtosecond transient absorption setup were given elsewhere ${ }^{14,15}$. The pump pulse energy was varied to look for the annihilation effect on the ground state recovery kinetics, and the probe wavelengths was set at $855 \mathrm{~nm}$ for B850 and at $880 \mathrm{~nm}$ for B875, respectively.

The $\left|\chi^{(3)}\right|$ reference for the DFWM is $\mathrm{CS}_{2}$ with its $\left|\chi^{(3)}\right|$ of $4 \times 10^{-13}$ esu at $1064 \mathrm{~nm}^{16,17}$, and $n^{\prime}$ s for the BChla containing samples were approximated by $n$ of the corresponding solvents $\left(n_{\mathrm{TTF}}=1.4070\right.$ and $\left.n_{\text {water }}=1.3330\right)$ because of low concentrations of BChla. Using our DFWM setup, only the magnitude, not the phase of the $\chi^{(3)}$ is obtained. The average molecular second hyperpolarizability $\langle\gamma\rangle$ in an isotropic medium was obtained by,

$$
\chi^{(3)}=\chi^{(3)}{ }_{s(v)}+10^{-6} L^{4} A<y>C
$$

where slvt stands for solvent, $A$ is Avogadro's number and $C$ is the concentration of the solute in mM. Thus, $\langle\gamma\rangle$ of the solute can be extracted from the slope of the $\chi^{(3)} v s$. C plot. 


\section{THEORETICAL CALCULATION RESULTS FOR (SiPcO) ${ }_{n}$ AND THEIR RELEVANCE TO $\langle\gamma\rangle$ OF BChla ARRAYS IN LH PROTEINS}

In the off-resonant regime, the molecular second order hyperpolarizability tensor $\gamma_{k j h}\left(\omega_{\sigma}, \omega_{1,}, \omega_{2}, \omega_{3}\right)$ can be described by the conventional sum-over-states (SOS) expression ${ }^{18}$ :

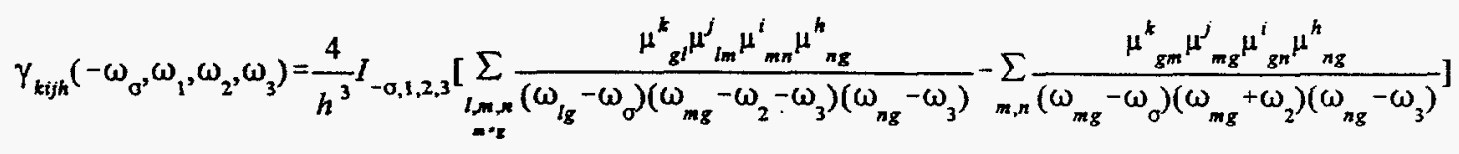

where $\mu_{a b}^{c}$ represents the cth cartisian component of the transition dipole moment between molecular states a and $\mathrm{b}$ and $\omega_{a b}$, the transition frequency for $a \rightarrow b$ transition. The operator $I_{-0,1,2,3}$ denotes that the expression must be averaged over all permutations of the frequencies $-\omega_{0}, \omega_{1}, \omega_{2}$, and $\omega_{3}$ with the cartisian components $k, j, i$, and $h$ permuted simultaneously. The first summation involves those two-photon allowed states, referred to type II terms, and the second term involves four photon volleys between the ground and one-photon allowed states, referred as type I terms. All the excitation are virtual here without significant population depletion of the ground state. The key to obtain a precise $\gamma_{k j h}$ value is obtain the energy levels and the transition dipoles in the equation (2). Because of the complexity of the structures for the macrocyles, semi-empirical methods with simplified energy levels must be employed. Because the laser frequencies are closer to those virtual transitions with the lowest energies and those transitions are often affected by intermacrocyle $\pi-\pi$ interactions, our recent calculations on (SiPcO) ${ }_{n}$ are based on the Frenkel exciton model for a chain of three level molecules, the ground state $\mid G>$, one-photon allowed state $\mid Q>$ and two-photon allowed state $|T\rangle$. Using a simplified analysis in the static and near-resonant regimes we identified two mechanisms which lead to enhancements in the dimer or trimer value of $\langle\gamma\rangle$ over that of the monomer. The first mechanism is a disruption of the balance between type I and type II terms in the sum over states expression for the second hyperpolarizability tensor $\gamma_{k i j h}$ caused by weak intermacrocycle interactions. The second is a near-resonance enhancement of the type II terms due to an intermacrocycle interaction induced shift in the monomer derived two-photon allowed states towards twice the laser photon energy. This analysis is in good agreement with the experimental results. The calculations suggest that the first mechanism is responsible for the 25 -fold monomer to dimer enhancement measured in this system and that the additional 4-fold enhancement found in going from the dimer to the trimer is primarily the result of the second mechanism. A complete description of the calculation can be found elsewhere ${ }^{5}$.

Although BChla and SiPcO are different molecular systems and the molecular aggregates in LH1/LH2 and in (SiPcO) have different configurations, the results for the latter are still relevant to the former in terms of the effect of the intermacrocycle interactions on $\langle\gamma\rangle$ enhancement. A more significant enhancement suggests a more altered energy levels from those of the monomer due to stronger intermacrocycle interactions.

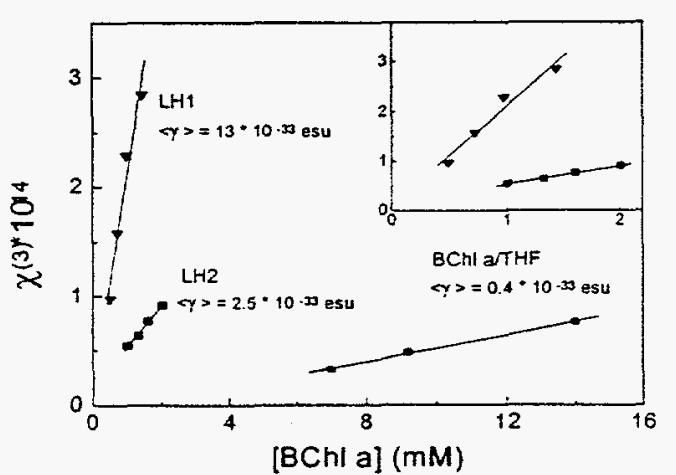

Figure 2. $\chi^{(3)}$ as functions of $\mathrm{BChla}$ concentration in THF, LH1 and LH2. Inset shows the enlarged low concentration region. The $\langle\gamma\rangle$ values are before correction for sphaeroidene. The $\langle\gamma\rangle$ value for $\mathrm{LH} 2$ is the average value for both $\mathrm{B} 800$ and $\mathrm{B} 850$ (see text).

\section{EXPERIMENTAL RESULTS}

\section{a. $\langle\gamma>$ of BChl a in Different Systems}

Figure 2 depicts the concentration dependence of $\chi^{(3)}$ for BChla in THF, LH1 and LH2. According to Equation (1),$\langle\gamma\rangle$ of $\mathrm{BChl} a$ was extracted from the slopes of the plots, to be $0.4 \times 10^{-33}$, $13.3 \times 10^{-33}$, and $2.5 \times 10^{-33} \mathrm{esu} / \mathrm{BChl} a$ for BChla in THF, LH1 and $\mathrm{LH} 2$, respectively. Based on the measurement conducted under the same conditions, the $\langle\gamma\rangle$ of BChla in LH1 and LH2 after the correction for the contribution of sphaeroidene (a molecule with a similar structure as $\beta$-carotene) attached to the protein are $13.0 \times 10^{-33}$ and $2.3 \times 10^{-33}$ esu/BChla, respectively -33 and 6 fold increases compared to that in THF. The $\langle\gamma\rangle$ for the BChla in B875 of LH1 is 5 times of that in $\mathrm{B} 850$ of $\mathrm{LH} 2$ for two reasons. First, LH2 contains both $\mathrm{B} 800$ and $\mathrm{B} 850$ arrays. $\mathrm{B} 800$ has a monomeric arrangement for $\mathrm{BChl}$, and the average $\mathrm{Mg}-\mathrm{Mg}$ distance between two adjacent $\mathrm{BChla}$ 
is $21.2 \AA^{6}$, too far for strong electronic interactions. Thus, $\langle\gamma\rangle$ of BChla in this array should be similar to that in monomeric $\mathrm{BChla}$ in THF. Consequently, the measured phase conjugated signal from LH2 is predominantly from B850. If we assume that the $\langle\gamma\rangle$ value for BChla in B800 of LH2 is the same for BChla in THF, and subtract it from the total $\langle\gamma\rangle$ for LH2, the $\langle\gamma\rangle$ for the BChla in $\mathrm{B} 850$ array would be $3.3 \times 10^{-33} \mathrm{esu} / \mathrm{BChl}$. This $\langle\gamma\rangle$ value is still four times lower than that of BChla in B875. Second, the face-to-face interactions between two adjacent BChla is more favorable in LH1 than in LH2 due to the diameter difference between LH1 and LH2 complexes (see Figure 1). The diameter of the B875 array in LH1 is $92 \AA^{7}$, which is much larger than $52 \AA$ for $\mathrm{B} 850$ array in LH2 ${ }^{6}$. As 32 and $18 \mathrm{BChla}$ are arranged symmetrically around $\mathrm{B} 875$ and $\mathrm{B} 850$ arrays, the average center-to-center distances between two adjacent $\mathrm{BChl} a$ are similar, but the angles between two adjacent macrocycle planes are considerably less in the larger array. Therefore, the $\pi-\pi$ interaction between two adjacent BChl $a$ 's could be stronger in $\mathrm{B} 875$ than in $\mathrm{B} 850$ resulting in a higher $\langle\boldsymbol{\gamma}\rangle$.

\section{b. Excited State $\langle\gamma\rangle$ of BChla in LH1 and LH2}

In the excited state nonresonant DFWM experiment, the pulse used to generate an excited state population will not participate in forming transient gratings, which results in the phase conjugated signal. The effect of the laser excitation is to create a reference state that is the excited state rather than the ground state.

We used a laser pulse at $583 \mathrm{~nm}$ to induce a $S_{0}-S_{2}\left(Q_{x}\right)$ transition. The initially populated $S_{2}$ state decays to $S_{1}$ state via internal conversion on a time scale of less than $1 \mathrm{ps}^{19}$. Samples with low BChla concentrations of $0.14 \mathrm{mM}$ (in LH1) and 0.44 $\mathrm{mM}$ (in LH2) were used, such that the phase conjugated signals in the absence of a $583 \mathrm{~nm}$ pulse were mainly from the solvent (see Figure 3). The signal increases with 583nm excitation are shown in Figure 3. From the fractions of excited BChla in the samples, $\langle\gamma\rangle$ values for the $\mathrm{S}_{1}$ state of $\mathrm{BChl} a$ are $7 \times 10^{-31}$ and $4 \times 10^{-31}$ esu/BChl $a$ in LH1 and LH2, a factor of 50 and 160 enhancement compared to $\langle\gamma\rangle$ of the ground state. The origin of the $\langle\gamma\rangle$ enhancement is not completely clear, because the uncertainly in energy levels of upper excited states as well as the transitions from $S_{1}$ state to those states.

Time dependence of the excited state phase conjugated signals for BChla in LH1 and LH2 is depicted by Figure 4. Because the increase of the excited state $\langle\gamma\rangle$ is mainly due to changing the reference state from the ground state to one of the excited states, the time dependence of the phase conjugated signals is expected to follow the excited state population decay (affected by energy transfer quenching and singlet-singlet annihilation). We do not intend to establish the excited state dynamics from the phase conjugated signal with the $583 \mathrm{~nm}$ excitation here, but merely compare our observation with transient absorption results for the same molecular systems under similar excitation conditions. In Figure $4 \mathrm{~b}$, the phase conjugated signal for LH2 has two time constants of $40 \mathrm{ps}$ and $0.9 \mathrm{~ns}$, which correspond well to the transient absorption results with
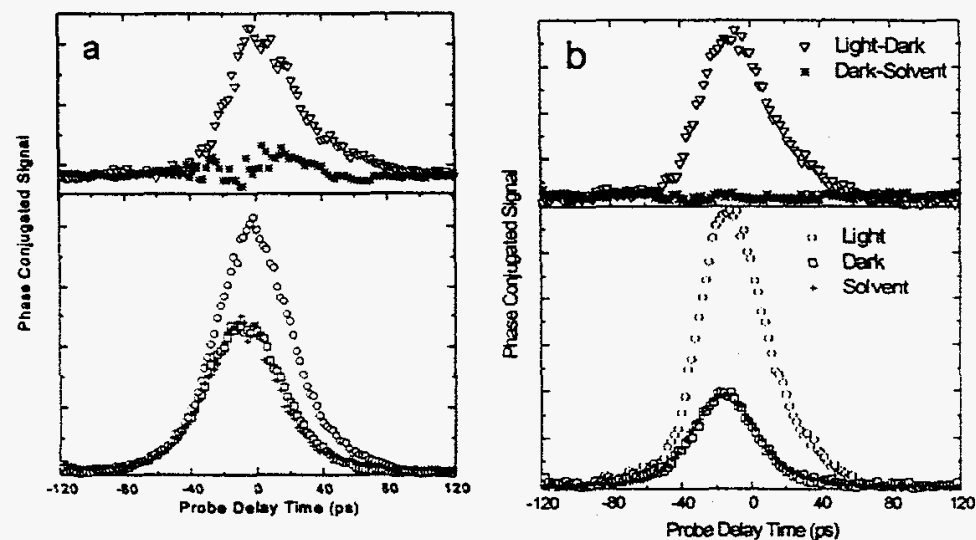

Figure 3. Phase conjugated signals (when $583 \mathrm{~nm}$ pulse overlaps with $1064 \mathrm{~nm}$ pump pulses) with and without $583 \mathrm{~nm}$ excitation as functions of the $1064 \mathrm{~nm}$ probe time for (a) $0.14 \mathrm{mM} \mathrm{BChla} \mathrm{in} \mathrm{LH1}$ and (b) $0.44 \mathrm{mM} \mathrm{BChla}$ in LH2. The bottom panels display the raw data, and the top panels show the signals from pure solute with and without $583 \mathrm{~nm}$ pulses. two time constants of $52 \mathrm{ps}$ and $0.948 \mathrm{~ns}$ when probing at $855 \mathrm{~nm}$. While the $0.9 \mathrm{~ns}$ component corresponds to the singlet excited state decay of BChl $a$ in $\mathrm{LH} 2^{20}$, the faster component could result from singlet-singlet annibilation in aggregates of LH2. The numbers of photons in each laser pulse of $583 \mathrm{~nm}$ light in both cases are approaching the annihilation limit with average 1.4 photons $/ \mathrm{LH} 2$ in DFWM and 1 photons/LH2 in the transient absorption experiments.

The interpretation of the phase conjugated signal for LH1 is puzzling. At an average of 4 photons/LH1 in each laser pulse, the phase conjugated signal can be fit by one exponential decay with time constant of $350 \mathrm{ps}$; while at 1 photons $/ \mathrm{LH} 1$ in each laser pulse, the single exponential decay time constant is $760 \mathrm{ps}$ (with a large uncertainty because of limited time range of the data). In comparison. the time constants of ground state recovery by our transient absorption measurements of $\mathrm{LH} 1$ gives a single exponential fit with a time constant of 0.8 ns when 
exciting with 0.3 photons $/ \mathrm{LH} 1$ in each laser pulse at $583 \mathrm{~nm}$. Using 3 photons/LH1 in each laser pulse at $583 \mathrm{~nm}$ as the excitation source, the transient absorption decay gives an additional 3 ps component due to singlet-singlet excitation annihilation. The 40-50ps component observed in LH2 was absent for LH1. This could be explained by an assumption of energy transfer/singlet-singlet annihilation between LH2 units in a super aggregates, which formed more readily than an aggregate of LH1 in the membrane.

\section{DISCUSSIONS}

\section{a. Origin of the $\langle\gamma\rangle$ Enhancement in LH1 and LH2}

Observed in our previous DFWM measurements on $\mu$-oxo SiPcO, $\langle\gamma\rangle$ for each macrocyclic unit is enhanced by a factor of 12 in the dimer and a factor of 33 in the trimer compared to that of the monomer ${ }^{5.6}$. This result provides a correlation between the $\langle\gamma\rangle$ enhancement and the $\pi-\pi$ interactions in the direction that is perpendicular to the macrocycle planes. Comparing $\langle\gamma\rangle$ of monomeric BChla in THF with that in B875 array of LH1 and B850
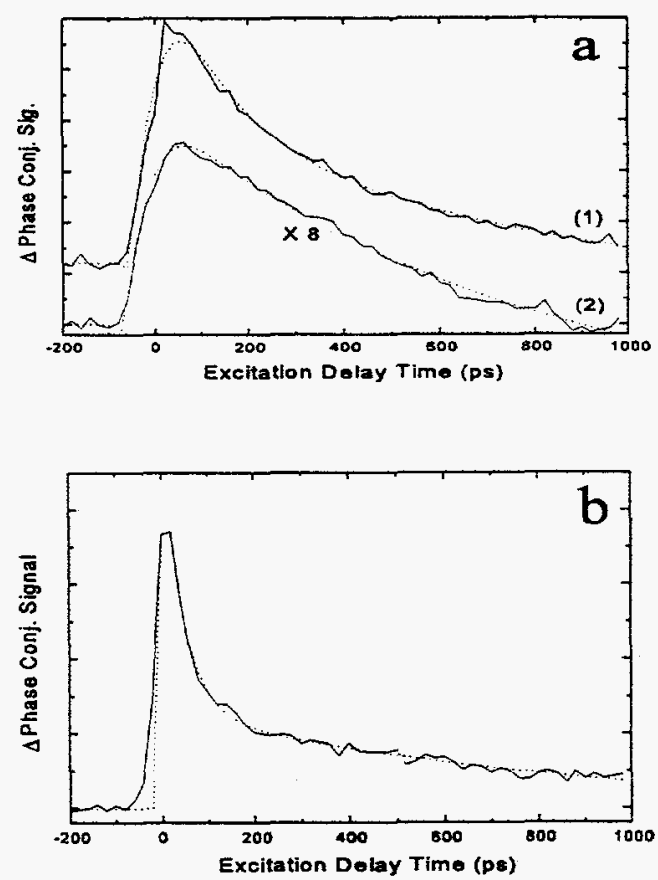

Figure $4.583 \mathrm{~nm}$ excitation delay time dependence of the phase conjugated signal in (a) LH1, with pulse energy of $250 \mu \mathrm{J}(1)$, and $60 \mu \mathrm{J}(2)$, and in (b) LH2 with pulse energy of $250 \mu \mathrm{J}$. Curve (1) and (2) in (a) were fit by a single exponential decay with a time constant of $350 \pm 50 \mathrm{ps}, 756 \pm 14$ ps, respectively. The curve in (b) was fit by a sum of two exponentials with coefficients of 0.27 and 0.73 and time constants of $943 \pm 79 \mathrm{ps}$ and $44 \pm 3$ ps. array of LH2, we observed $\langle\gamma\rangle$ enhancement again when BChla macrocycles are arranged into a circle with tilted "face-to-face" configurations. In cases of BChla in B875 and B850 arrays, the $\langle\gamma\rangle$ for each BChla is 33 and 8 times of that for monomeric BChla in THF, respectively.

Because of the differences in monomer structures and in the configuration of the molecular aggregates between $\mu$-oxo SiPc and $\mathrm{BChl} a$ in B875 and B850, it is difficult to compare different systems directly and to identify the one that provides a higher $\langle\gamma\rangle$ enhancement. At this point, we have not modeled $\langle\gamma\rangle$ for a "face-to-face" molecular aggregate as functions of structural parameters; so any conclusion regarding a preferred configuration for an optimized $\langle\gamma\rangle$ is premature. Nevertheless, a factor of four increase in $\langle\gamma\rangle$ for $\mathrm{BChl} a$ in B875 than that in B850 was observed despite similarities in the configurations of protein-bound $\mathrm{BChl} a$ arrays. Although, one could relate the progressive increase of the $\chi^{(3)}$ in B800, $\mathrm{B} 850$ and $\mathrm{B} 875$ by the near resonant one-photon effect that is due to an increasing closeness of the peak frequencies to the laser frequency, this effect alone cannot explain the enhancement factors observed in our experiments. According to the sum-over-state (SOS) expression ${ }^{18}$, the enhancement due to this near resonant effect can be estimated to be 1.7 and 2.1 for B850 and B875 compared to B800, much smaller than 8 and 33 observed in the experiment. In our studies of $\langle\gamma\rangle$ enhancement in $\mu$-oxo SiPc "face-to-face" oligomers", two mechanisms for the $\langle\gamma\rangle$ enhancement were proposed: (1) a shift of a two-photon allowed state energy toward to the second harmonic of the laser frequency, and (2) a shift of a charge transfer band toward to laser frequency, both were due to inter-macrocycle $\pi-\pi$ interactions when SiPcO oligomers were formed with the "face-toface" configuration. Our study has indicated that these shifts due to intermacrocycle interactions can cause a substantial enhancement of $\langle\gamma\rangle$ when the monomer $\langle\gamma\rangle$ has a well balanced two- and one-photon terms in the SOS expression. If we attribute the $\langle\gamma\rangle$ enhancement of $\mathrm{BChl} a$ in $\mathrm{B} 850$ and B875 similarly to the mechanism (1) or (2), the difference in $\langle\gamma\rangle$ values for BChla in B875 and B850 implies a stronger interaction in the former, resulting from a better "face-to-face" overlap between the macrocycles. Because average center-tocenter distances between the adjacent $\mathrm{BChl} a$ in $\mathrm{B} 850$ and $\mathrm{B} 875$ only differ by $0.05 \AA^{6.7}$, the stronger interaction between macrocycles must come from a pair-wise arrangement in B875. This suggestion agrees with a recent result of hole-burning experiment indicating a stronger interaction between the $\mathrm{BChla}$ in $\mathrm{B} 875$ than in $\mathrm{B} 850^{21}$. It is also worth mentioning that in a recent study of nonlinear absorption in $\mathrm{LH}_{2}{ }^{22}$, a giant $\chi^{(3)}$ was predicted from strong excitonic coupling within the circular aggregates of BChla. Further theoretical modeling of the $\chi^{(3)}$ enhancement in B875 and B850 has been planned in order to find its origin. 


\section{b. Implication of Excited State $\langle\gamma\rangle$ Enhancement in Stacked BChla}

A significant increase of $\langle\gamma\rangle$ for BChla in LH1 and LH2 has been observed through our DFWM measurement when an excitation pulse at $583 \mathrm{~nm}$ is applied. According to SOS expression of $\langle\gamma\rangle$ for the DFWM ${ }^{18}$, the $\langle\gamma\rangle$ for one- and twophoton processes are proportional to the products of the transition dipoles from the reference state (normally, the ground state) to the other stationary states and inversely proportional to the products of frequency differences between the laser and transitions from the reference state to the stationary states. Therefore, when an excited state is populated prior to a nonresonant $\chi^{(3)}$ measurement, this state becomes the reference state. During the lifetime of this excited state, the observed enhancement of $\langle\gamma\rangle$ could result from (1) a decrease in the energy gap between the laser frequency and the transition frequency from the populated excited state to other upper states and (2) increase in the transition dipole moments from the populated excited state to certain other higher states. In particular, for $\mathrm{BChl} a$, the enhancement due to $\mathrm{S}_{1}$ state population may result from increasing contributions of B states which is comparatively unimportant in the ground state $\langle\gamma\rangle$. However, there is not enough information on the upper state levels and transition dipoles to verify the precise mechanism of the excited state $\langle\gamma\rangle$ enhancement at this point.

The excited state dynamics of BChla in LH1 and LH2 involve multiple energy transfer and trapping processes have been studied extensively ${ }^{9,23,24}$. However, the details of those processes reflected in the excited state dynamics is not the focus here. Our preliminary results merely show that the phase conjugated signals of excited LH1 and LH2 have been enhanced which correspond to 50 and 160 fold increases in $\langle\gamma\rangle$ compared to that of the ground state BChla. The ratio of $\langle\gamma\rangle$ for BChla in LH1 and $\mathrm{LH} 2$ is much smaller in excited states than in the ground states, indicating the decrease in the energy gaps between the reference state and the virtual states is the main cause of the enhancement. At $583 \mathrm{~nm}$, the pump laser populates the $S_{2}$ state $\left(Q_{x}\right.$ excitation) initially, then the $S_{1}$ state is populated through a ultrafast internal conversion within $1 \mathrm{ps}^{19}$. The transition frequency from $S_{1}$ state to higher excited states could be close to that of photons at $1064 \mathrm{~nm}$, thus, significantly increasing the phase conjugated signal. This observation indicates a potential application in light controlled molecular devices.

\section{ACKNOWLEDGMENT}

LXC would like to acknowledge the support for this work by the U. S. Department of Energy, Office of Basic Energy Sciences, Division of Chemical Sciences (under Contract No. W-31-109-Eng-38). PDL acknowledges an appointment to the Alexander Hollaender Distinguished Postdoctoral Fellowship Program sponsored by the U. S. Department of Energy, Office of Health and Environmental Research. FCS and ESM would like to acknowledge the support from National Science Foundation through NSF-DMR9312029. We also like to thank Dr. David Tiede for his LH1 samples and Dr. Scott Greenfield for his help in measuring excited state kinetics for $\mathrm{BChla}$.

\section{REFERENCES}

1. P. N. Prasad, D. J. Williams, Introduction to Nonlinear Optical Effects in Molecules and Polymers (Wiley, New York, 1991).

2. D. S. Chemla, J. Zyss, Eds., Nonlinear Optical Properties of Organic Molecules and Crystals, Vol. 1 \& 2 (Academic Press, New York, 1987).

3. A. J. Heeger, J. Oreustein, D. R. Ulrich, Eds., Nonlinear Optical Properties of Polymers, Mat. Res. Soc. Symp. Proc., Vol. 109 (MRS: Pittsburgh, 1988).

4. $\quad$ L. X. Chen, B. K. Mandal, B. Bihari, A. K. Sinha, M. Kamath, SPIE Proc. 2527 (1995) 61.

5. E. S. Manas, F. C. Spano, L. X. Chen, J. Chem. Phys., in press.

6. G. McDermott, S. M. Prince, A. A. Freer, A. M. Hawthornthwaite-Lawless, M. Z. Papiz, R. J. Cogdell, N. W. Isaacs, Nature 374 (1995) 517.

7. S. Karrasch, P. A. Bullough, R. Ghosh, The EMBO Journal 14 (1995) 631.

8. A. Freer, S. Prince, K. Sauer, M. Papiz, A. Hawthornthwaite-Lawless, G. McDermott, R. Cogdell, N. Isaacs, Structure 4 (1996) 449.

9. R. van Grondelle, J. P. Dekker, T. Gillbro, V. Sundström, Biochim. Biophys. Acta 1187 (1994)1.

10. (a) J. R. Heflin, D. C. Rodenberger, R. F. Shi, M. Wu, N. Q. Wang, Y. M. Cai, A. F. Garito, Phys. Rev. A 45 (1992)

R4233; (b) D. C. Rodenberger, J. R. Heflin, A. F. Garito, Nature 359 (1992) 309.

11. L. X. Chen, P. D. Laible, Chem. Phys. Lett. 270 (1997) 255.

12. R. K. Clayton, B. J. Clayton, Proc. Natl. Acad. Sci. LSA 78 (1981) 5583. 
13. R. K. Clayton in Bacterial Photosynthesis, H. Gest, A. San Pietro and L. P. Vernon, eds., (1963) p.495 (Antioch Press, Yellow Springs).

14. S. R. Greenfield, M. Seibert, Govindjee, M. R. Wasielewski, J. Chem. Phys., B101, (1997) 2251.

15. S. R. Greenfield, M. R. Wasielewski, Opt. Lett. 259 (1995) 1394.

16. R. W. Hellwarth, Prog. Quant. Electr. 5 (1977) 1.

17. M. J. Moran, C.-Y. She, R. L. Carman, IEEE J. Quant. Electr. QE-11 (1975) 259.

18. B. J. Orr, J. F. Ward, Mol. Phys. 20 (1971) 513.

19. A. O. Ganago, E. P. Parker, P. D. Laible, A. C. Albrecht, T. G. Owens, Laser Physics, 5 (1995) 693.

20. P. Sebban, G. Jolchine, I. Moya, Photochem. Photobiol., 39 (1984) 247.

21. N. R. S. Reddy, H.-M. Wu, R. Jankowiak, R. Picorel, R. J. Cogdell, G. J. Small, Photosynthesis Res., 48 (1996) 277.

22. D. Leupold, J. Stiel, K. Teuchner, F. Nowak, W. Sander, B. Ücker, H. Scheer, Phys. Rev. Lett., 77 (1996) 4675.

23. S. E. Bradforth, R. Jimenez, F. van Mourik, R. van Grondelle, G. R. Fleming, J. Phys. Chem., 99 (1995) 16179.

24. (a) S. Hess, M. Chachisvilis, K. Timpman, M. R. Jones, G. J. S. Fowler, C. N. Hunter, V. Sunström, Proc. Nat. Acta. Sci., USA, 92 (1995) 12333; (b) M. Chachisvilis, T. Pullerits, M. R. Jones, C. N. Hunter, V. Sunström, Chem. Phys. Lett., 224 (1994) 345. 